\title{
Clinical impact of FDG PET-CT on management decisions for patients with primary biliary tumours
}

\author{
Raneem Albazaz • Chirag N. Patel • \\ Fahmid U. Chowdhury • Andrew F. Scarsbrook
}

Received: 25 January 2013 / Revised: 20 June 2013 / Accepted: 24 June 2013 / Published online: 25 July 2013

(C) The Author(s) 2013. This article is published with open access at Springerlink.com

\begin{abstract}
Objectives To assess the impact on clinical management of introducing ${ }^{18} \mathrm{~F}$-fluoro-2-deoxy-D-glucose (FDG) positron emission tomography (PET)-computed tomography (CT) in to the work-up of patients with primary and recurrent biliary malignancy.

Methods Consecutive patients with primary biliary tumours undergoing FDG PET-CT at a single large tertiary referral centre between November 2007 and September 2010 were retrospectively analysed. Findings on FDG PET-CT compared with $\mathrm{CT} /$ magnetic resonance imaging (MRI) and impact on subsequent patient management were evaluated. Impact was divided into: (1) major-detection of occult disease or characterisation of indeterminate lesion(s) on CT/MRI; (2) minor - confirmation of suspected metastases seen on CT/MRI; (3) no impact.

Results One hundred and eleven patients underwent 118 FDG PET-CT scans, including 30 with suspected gallbladder carcinoma and 81 with cholangiocarcinoma. Eighty-nine scans were performed for initial staging, five for restaging following neoadjuvant chemotherapy and 24 for suspected disease recurrence. In 33 cases (28 \%), FDG PET-CT had a major impact on subsequent patient management (39\% gallbladder carcinoma, $26 \%$ intrahepatic cholangiocarcinoma and $21 \%$ extrahepatic cholangiocarcinoma). FDG PET-CT had a minor impact in 20 cases (17\%) and no impact in 65 cases $(55 \%)$.
\end{abstract}

\section{R. Albazaz ( $\square)$}

Department of Radiology, Level 0, Bexley Wing, St James's University Hospital, Beckett Street, Leeds LS9 7TF, UK

e-mail: raneemalbazaz@hotmail.com

C. N. Patel • F. U. Chowdhury • A. F. Scarsbrook Department of Nuclear Medicine, St James's University Hospital, Beckett Street, Leeds LS9 7TF, UK
Conclusions By detecting occult metastatic disease and characterising indeterminate lesions, FDG PET-CT can have a major influence on clinical decision-making in primary and recurrent biliary malignancy.

Keywords Gallbladder carcinoma - Cholangiocarcinoma . Biliary tumours $\cdot{ }^{18}$ F-fluoro-2-deoxy-D-glucose positron emission tomography-computed tomography

Teaching Points:

- FDG PET-CT influenced management in a significant number of patients with biliary malignancy.

- FDG PET-CT is effective at detecting occult metastases and characterising indeterminate lesions.

- FDG PET-CT helps triage patients with biliary malignancy to the correct treatment.

\section{Introduction}

Primary neoplasms of the biliary system are a heterogeneous group largely comprising of gallbladder carcinoma and cholangiocarcinoma with a few other much rarer tumours. Traditionally these tumours have a high mortality rate, with surgical excision being the mainstay of long-term survival [1]. Advances in surgery have increased the likelihood of achieving local disease control and now limited distant spread may also be considered for resection. Accurate staging is critical for this to occur, not only to aid surgical planning but also to prevent unnecessary surgical intervention in those with advanced disease.

Despite pre-operative assessment with traditional crosssectional imaging techniques, up to a third of patients with primary biliary malignancy still undergo unnecessary 
laparotomy [2]. The main contraindications to resectability found at surgery are occult peritoneal and liver metastases, and, less commonly, vascular and lymph node invasion [3, 4]. These may not be detected due to inability of conventional imaging modalities (computed tomography $[\mathrm{CT}] /$ magnetic resonance imaging [MRI]) to accurately distinguish between benign and malignant processes based on size or anatomical criteria alone or when prior intervention distorts normal anatomy. Gallbladder carcinoma in particular is associated with more frequent early dissemination, whereas cholangiocarcinoma is likely to be locally invasive and has a slightly longer survival [2].

${ }^{18}$ F-Fluoro-2-deoxy-D-glucose (FDG) integrated positron emission tomography (PET)-computed tomography (CT) is a non-invasive imaging technique that allows in vivo assessment of the metabolic processes underlying malignant disease. It has been shown to be more accurate than CT/MRI in the assessment of several primary and recurrent tumours, including colorectal, lung and oesophageal cancer [5-7]. Moreover, it is reported to have a consistent impact on intended management that is not restricted to cancer type [8]. By providing additional functional information and in the correct setting, FDG PET-CT can allow earlier detection of tumours, identification of occult metastatic disease, characterisation of indeterminate lesions, assessment of therapeutic response and overall more accurate staging for potential resection.

Several previous studies have assessed the ability of FDG PET-CT to detect primary biliary tumours; however, few report its overall efficacy in the management pathway of patients with primary biliary malignancy. The aim of this study was to assess the impact on clinical management of introducing FDG PET-CT in to the work-up of patients with potentially resectable primary biliary malignancy and in those undergoing FDG PET-CT for response assessment following chemotherapy or with suspected disease recurrence.

\section{Materials and methods}

\section{Patients}

Consecutive patients with primary biliary tumours undergoing FDG PET-CT at a single large tertiary referral centre over a 35 -month period were retrospectively analysed (November 2007 to September 2010). Formal ethics committee approval was not required at our institution for a case review of this kind. Data were accessed from the institutional radiology information system (CRIS; Healthcare Software Systems, Banbury, UK) and Patient Pathway Manager (PPM, Leeds, UK; an electronic patient record system). Referral for FDG PET-CT was made on a case-by-case basis after discussion at a specialist multidisciplinary team (MDT) meeting. The main indication for performing FDG PET-CT was for initial staging to determine suitability for surgical resection where there was some ambiguity on initial conventional imaging. The other indications were for restaging following neoadjuvant chemotherapy and for suspected disease recurrence. Patients with locally advanced disease or distant metastatic disease that was deemed unresectable on conventional imaging were referred for palliative chemotherapy where appropriate.

\section{Imaging}

Imaging was retrospectively reviewed independently by two experienced radiologists. Both readers are dual trained in Radiology and Nuclear Medicine and respectively have 13/11 years of experience in CT and MRI and 9/7 years of experience in PET-CT. Prior radiology reports were subsequently reviewed but not at the time of image analysis. Imaging, particularly that performed externally, was validated and accepted as consistent for diagnosis at time of MDT review. If imaging was deemed suboptimal for diagnosis, then it was repeated at our institution. Any discrepancies were discussed in order to reach a consensus view.

Imaging for each patient was retrieved from the institutional picture archiving and communications system (PACS) (IMPAX; AGFA Healthcare, Mortsel, Belgium). Contrastenhanced CT (CECT) thorax/abdomen/pelvis was performed in 76 cases. The mean time between the previous CECT and the PET-CT was 38 days (range 1-125 days, lower quartile 22 days and upper quartile 46 days). Most cases were scanned within a 90-day period with only four, all cholangiocarcinomas, scanned outside this time. CECT was performed at our institution $(n=57)$ after referral from the regional oncology team on either a 16- or 64-slice scanner (Siemens Healthcare, Erlangen, Germany) using a contiguous 3-mm reconstruction and following a bolus of $100 \mathrm{ml}$ iodinated contrast medium (Omnipaque $300 \mathrm{mg} \mathrm{I} / \mathrm{ml}$; GE Healthcare, Oslo, Norway). The remaining CTs had been acquired at one of several referring hospitals on a range of different scanner types using similar acquisition parameters $(n=18)$.

Liver MRI was performed in 72 cases. The mean time between the previous MRI and the PET-CT was 15 days (range 1-88 days, lower quartile 3 days and upper quartile 21 days). MRI was performed at our institution in 67 of the 72 cases on a 1.5-T system (Symphony/Avanto; Siemens Healthcare, Erlangen, Germany) using a body phased array coil and a breath-holding technique. T1/T2/T2*-weighted sequences were obtained with the use of intravenous gadolinium (Gadovist; Bayer Healthcare Pharmaceuticals, Berlin, Germany) and, where appropriate, liver-specific contrast agents (Resovist prior to its withdrawal in 2009 and Primovist thereafter; Bayer Healthcare Pharmaceuticals, Berlin, Germany). Diffusion-weighted imaging was also performed for each case with $b$ values of 50, 200, 500 and $750 \mathrm{~s} / \mathrm{mm}^{2}$. 
PET-CT scans were performed prior to 1 st June 2010 on a 16-slice Discovery STE PET-CT scanner (GE Healthcare, Amersham, UK), and thereafter on a 64-slice Philips Gemini TF64 scanner (Philips Healthcare, Amsterdam, Netherlands), 60 min following a $400-\mathrm{MBq}$ dose of intravenous FDG. Images were acquired from the skull base to upper thigh. The CT component of the PET-CT was performed according to a standardised protocol with the following settings: $140 \mathrm{kV}$; $80 \mathrm{mAs}$; tube rotation time $0.5 \mathrm{~s}$ per rotation; pitch 6 ; section thickness $3.75 \mathrm{~mm}$ (to match PET section thickness). Patients were asked to maintain normal shallow respiration during CT acquisition. No iodinated contrast material was administered. Imaging was reviewed on specialised PET-CT workstations (XD3; Mirada Medical, Oxford, UK).

\section{Analysis of clinical impact}

The clinical impact of FDG PET-CT on the management of patients was evaluated using the institutional oncological electronic patient record system (Patient Pathway Manager), which prospectively documents MDT decisions based on discussion between medical and radiation oncologists, surgeons, radiologists and pathologists. Information was also obtained from the institution's radiology information system (CRIS). Follow-up information was obtained for a minimum 12-month period subsequent to the date of the PET-CT scan. All PET-CT findings were correlated with the results of histological, radiological and clinical follow-up. A hypermetabolic FDG lesion was considered truly positive for involvement if malignancy was proven by histological analysis or if the lesion progressed on follow-up imaging or resolved after therapy. An FDG-negative lesion was considered truly negative if its size remained stable on imaging follow-up.

The impact of FDG PET-CT in addition to CT/MRI findings on the management of patients was assessed with respect to potential change of management by either the identification of distant unresectable disease or confirmation of its absence. Three categories of impact were considered:

Major impact:

- Evidence of unresectable metastatic disease on FDG PET-CT, which was either indeterminate or evaded detection on prior CT/MRI.

- Additional FDG PET-CT findings that would extend the surgical field.

- FDG PET-CT characterisation of indeterminate lesions on $\mathrm{CT} / \mathrm{MRI}$ as benign, allowing curative resection to be attempted.

Minor impact:

- FDG PET-CT confirmation of suspicious unresectable findings on $\mathrm{CT} / \mathrm{MRI}$.
No impact:

- No additional findings on FDG PET-CT compared with CT/MRI.

- Indeterminate findings on FDG PET-CT which did not alter the status of the patient with regards to resectability.

If findings on FDG PET-CT fell into both a minor impact and a major impact category then overall the impact was considered as major.

\section{Results}

In total, 111 patients underwent 118 FDG PET-CT scans (45 men, 66 women). Mean patient age was 65 years (range 24 87 , lower quartile 58 and upper quartile 73 ). There were 30 patients with suspected gallbladder carcinoma and 81 with cholangiocarcinoma (47 intra-hepatic, 34 extra-hepatic). Eighty-nine scans were performed for initial staging (Table 1), five scans for restaging following chemotherapy prior to consideration of radical surgery and 24 scans for assessment of suspected disease recurrence (Table 2).

The majority of patients underwent initial staging with CECT thorax/abdomen/pelvis with or without MRI. A minority only underwent MRI of the upper abdomen prior to PET-CT; however, in none of these patients did PET-CT have an impact by finding disease outside of the imaging field performed for MRI.

Overall, FDG PET-CT had a major impact on subsequent patient management in 33 cases $(28 \%)$. This consisted of $39 \%(n=12)$ of gallbladder carcinomas, $26 \%(n=14)$ of intra-hepatic cholangiocarcinomas and $21 \%(n=7)$ of extrahepatic cholangiocarcinomas. Eleven of these patients had occult metastatic disease: sites included liver $(n=4)$, peritoneum $(n=6)$ and bone $(n=1)$. There were seven cases of unsuspected lymph node involvement; four unsuspected lymph node involvement and peritoneal disease; two unsuspected liver metastases and lymph node involvement; two were found to have unsuspected synchronous primary cancers. In a further seven patients, FDG PET-CT clarified indeterminate lesions seen on cross-sectional imaging as either malignant $(n=2)$ or benign $(n=5)$. FDG PET-CT had a minor impact in $17 \%$ of cases $(20 / 118)$ and no impact in $55 \%$ of cases (65/118). These results are summarised in Table 3, with a breakdown of the impact of FDG PET-CT performed for initial staging versus suspected disease recurrence.

\section{Gallbladder carcinoma}

Thirty patients with suspected gallbladder carcinoma underwent a total of 31 FDG PET-CT scans. Twenty-seven were performed for initial staging and four for suspected disease recurrence. 
Table 1 PET-CT findings in comparison to CT/MRI for scans performed for initial staging and re-staging following chemotherapy. The findings on PET-CT compared with findings on $\mathrm{CT} / \mathrm{MRI}$ are presented as a fraction of the total number of cases in each category

$G B C$ gallbladder carcinoma, $C C$ cholangiocarcinoma

\begin{tabular}{llllll}
\hline $\begin{array}{l}\text { CT/MR imaging for disease } \\
\text { resectability at initial staging }\end{array}$ & $\begin{array}{l}\text { PET-CT } \\
\text { negative }\end{array}$ & $\begin{array}{l}\text { PET-CT } \\
\text { indeterminate }\end{array}$ & $\begin{array}{l}\text { PET-CT } \\
\text { positive }\end{array}$ & $\begin{array}{l}\text { PET-CT } \\
\text { false-negative }\end{array}$ & $\begin{array}{l}\text { PET-CT } \\
\text { false-positive }\end{array}$ \\
\hline $\begin{array}{l}\text { Resectable } \\
\text { GBC }\end{array}$ & $5 / 27$ & $0 / 27$ & $8 / 27$ & $3 / 27$ & $0 / 27$ \\
CC & $37 / 67$ & $2 / 67$ & $10 / 67$ & $3 / 67$ & $1 / 67$ \\
$\quad$ Total & $42 / 94$ & $2 / 94$ & $18 / 94$ & $6 / 94$ & $1 / 94$ \\
Indeterminate & & & & & \\
GBC & $0 / 27$ & $1 / 27$ & $1 / 27$ & $0 / 27$ & $1 / 27$ \\
CC & $2 / 67$ & $0 / 67$ & $1 / 67$ & $0 / 67$ & $0 / 67$ \\
$\quad$ Total & $2 / 94$ & $1 / 94$ & $2 / 94$ & $0 / 94$ & $1 / 94$ \\
Suspected unresectable & & & & & \\
$\quad$ GBC & $0 / 27$ & $0 / 27$ & $8 / 27$ & $0 / 27$ & $0 / 27$ \\
CC & $0 / 67$ & $0 / 67$ & $10 / 67$ & $1 / 67$ & $0 / 67$ \\
Total & $0 / 94$ & $0 / 94$ & $18 / 94$ & $1 / 94$ & $0 / 94$ \\
\hline
\end{tabular}

FDG PET-CT had a major impact on patient management in $39 \%$ of cases (12/31), including finding disease recurrence in two, characterising indeterminate peritoneal nodules as benign in one, and finding new disease sites in seven (retroperitoneal lymph node involvement $n=1$; lymph node and liver/peritoneal metastases $n=2$ (Fig. 1); liver/peritoneal metastases $n=4)$. Synchronous primary cancers were found incidentally in two patients (gastric and rectal). Overall, FDG PET-CT upstaged $26 \%$ (7/27) of the scans performed for initial staging and downstaged a further $4 \%(1 / 27)$.

FDG PET-CT had a minor impact on patient management in $6 \%$ of cases $(2 / 31)$ by confirming the presence of suspicious unresectable peritoneal disease in one case and liver metastases in the other, as suspected on CT/MRI. FDG PET-CT had no impact on patient management in the remaining $55 \%$ of cases (17/31).

FDG PET-CT was falsely negative in three cases for peritoneal involvement that was later found at surgery.
FDG PET-CT was falsely positive in one patient with a suspected malignant gallbladder mass, where the final histology showed xanthogranulomatous cholecystitis only.

Of the 26 confirmed gallbladder carcinomas, only 15 remained in situ at time of PET-CT. All showed uptake of FDG (SUV mean 15.2, range 4.0-38.4; sensitivity $100 \%$ ).

\section{Cholangiocarcinoma}

Eighty-one patients with suspected cholangiocarcinoma (47 intra-hepatic, 34 extra-hepatic) underwent a total of 87 FDG PET-CT scans. Sixty-two scans were performed for initial staging (39 intra-hepatic, 23 extra-hepatic), 20 for suspected disease recurrence ( 9 intra-hepatic, 11 extra-hepatic) and 5 for restaging following chemotherapy prior to consideration of radical surgery (4 intra-hepatic, 1 extra-hepatic).

FDG PET-CT had a major impact on patient management in $24 \%$ of cases $(21 / 87)$. Of these, $26 \%$ were intra-hepatic
Table 2 PET-CT findings in comparison to CT/MRI for scans performed for possible recurrence. The findings on PET-CT compared with findings on $\mathrm{CT} /$ MRI are presented as a fraction of the total number of cases in each category

$G B C$ gallbladder carcinoma, $C C$ cholangiocarcinoma

\begin{tabular}{llllll}
\hline $\begin{array}{l}\text { CT/MR imaging for } \\
\text { suspected recurrence }\end{array}$ & $\begin{array}{l}\text { PET-CT } \\
\text { negative }\end{array}$ & $\begin{array}{l}\text { PET-CT } \\
\text { indeterminate }\end{array}$ & $\begin{array}{l}\text { PET-CT } \\
\text { positive }\end{array}$ & $\begin{array}{l}\text { PET-CT } \\
\text { false-negative }\end{array}$ & $\begin{array}{l}\text { PET-CT } \\
\text { false-positive }\end{array}$ \\
\hline Clear & & & & & \\
GBC & $1 / 4$ & $0 / 4$ & $2 / 4$ & $0 / 4$ & $0 / 4$ \\
CC & $1 / 20$ & $0 / 20$ & $3 / 20$ & $0 / 20$ & $0 / 20$ \\
Total & $2 / 24$ & $0 / 24$ & $5 / 24$ & $0 / 24$ & $0 / 24$ \\
Indeterminate & & & & & $0 / 4$ \\
GBC & $1 / 4$ & $0 / 4$ & $0 / 4$ & $0 / 4$ & $0 / 20$ \\
CC & $2 / 20$ & $0 / 20$ & $2 / 20$ & $1 / 20$ & $0 / 24$ \\
Total & $3 / 24$ & $0 / 24$ & $2 / 24$ & $1 / 24$ & $0 / 4$ \\
Suspected & & & & & $0 / 20$ \\
GBC & $0 / 4$ & $0 / 4$ & $0 / 4$ & $0 / 4$ & $0 / 24$ \\
CC & $0 / 20$ & $0 / 20$ & $11 / 20$ & $0 / 20$ & \\
Total & $0 / 24$ & $0 / 24$ & $11 / 24$ & $0 / 24$ & \\
\hline
\end{tabular}


Table 3 Clinical impact of PET-CT

\begin{tabular}{lllll}
\hline & & Major & Minor & No impact \\
\hline GB & Initial staging & $33 \%(9 / 27)$ & $7 \%(2 / 27)$ & $60 \%(16 / 27)$ \\
& Suspected recurrence & $75 \%(3 / 4)$ & $0 \%(0 / 4)$ & $25 \%(1 / 4)$ \\
& Total & $39 \%(12 / 31)$ & $6 \%(2 / 31)$ & $55 \%(17 / 31)$ \\
CC intrahepatic & Initial staging & $20 \%(9 / 45)$ & $11 \%(5 / 45)$ & $69 \%(31 / 45)$ \\
& Suspected recurrence & $56 \%(5 / 9)$ & $44 \%(4 / 9)$ & $0 \%(0 / 9)$ \\
CC extrahepatic & Total & $26 \%(14 / 54)$ & $17 \%(9 / 54)$ & $57 \%(31 / 54)$ \\
& Initial staging & $23 \%(5 / 22)$ & $9 \%(2 / 22)$ & $68 \%(15 / 22)$ \\
CC All & Suspected recurrence & $18 \%(2 / 11)$ & $64 \%(7 / 11)$ & $5 \%(2 / 11)$ \\
& Total & $21 \%(7 / 33)$ & $10 \%(7 / 67)$ & $69 \%(46 / 67)$ \\
Total & Initial staging & $21 \%(14 / 67)$ & $55 \%(11 / 20)$ & $10 \%(2 / 20)$ \\
\hline
\end{tabular}

$G B C$ gallbladder carcinoma, $C C$ cholangiocarcinoma

(14/54) and $21 \%$ extra-hepatic (7/33). FDG PET-CT found disease recurrence at sites not suspected on CT/MRI $(n=5)$ and characterised four indeterminate lesions as malignant (pulmonary nodule $n=1$ ) or benign (pulmonary nodule $n=1$; peritoneal nodule $n=1$; peritoneal haziness/lymph node enlargement in a case of suspected recurrence $n=1$ ). FDG PET-CT confirmed response to chemotherapy in a patient with a large intra-hepatic cholangiocarcinoma and a bone metastasis that was unsuspected on CT/MRI (Fig. 2). FDG PET-CT found new disease sites in 11 (lymph node involvement $n=6$; liver and lymph node involvement $n=1$; peritoneal disease $n=2$; lymph node involvement and peritoneal disease $n=1$ [Fig 3]; bone metastasis $n=1$ ). Overall, FDG PET-CT upstaged $19 \%(12 / 62)$ and downstaged $3 \%(2 / 62)$ of the scans performed for initial staging (downstaged one peritoneal deposit and one pulmonary nodule, both in intrahepatic cholangiocarcinomas).

FDG PET-CT had a minor impact on patient management in $21 \%$ of cases $(18 / 87)$ by confirming suspicious unresectable findings seen on CT/MRI (9/54 of intra-hepatic and 9/33 of extra-hepatic cholangiocarcinomas). FDG PET$\mathrm{CT}$ had no impact on patient management in the remaining $55 \%$ of cases $(48 / 87)$.

FDG PET-CT was falsely negative in five cases. The first was a patient with primary sclerosing cholangitis (PSC) and a suspicious right liver mass on surveillance MRI, later confirmed to be a well-differentiated cholangiocarcinoma (no mucinous differentiation on histology). FDG PET-CT was also falsely negative in another PSC patient where CECT showed a filling defect in the distal CBD associated with biliary dilatation; surgery was undertaken based on the CECT findings and post-operative histology confirmed two small foci of cholangiocarcinoma measuring $8 \mathrm{~mm}$ and $13 \mathrm{~mm}$. FDG PET-CT was falsely negative for peritoneal disease in a patient with an FDG-avid intra-hepatic cholangiocarcinoma and in two patients with extra-hepatic cholangiocarcinomas (both primary tumours were also FDG negative).

FDG PET-CT was falsely positive in one patient found to have a hilar mass on MRI. FDG PET-CT showed uptake in the liver, mesenteric nodes and peritoneal deposits thought to
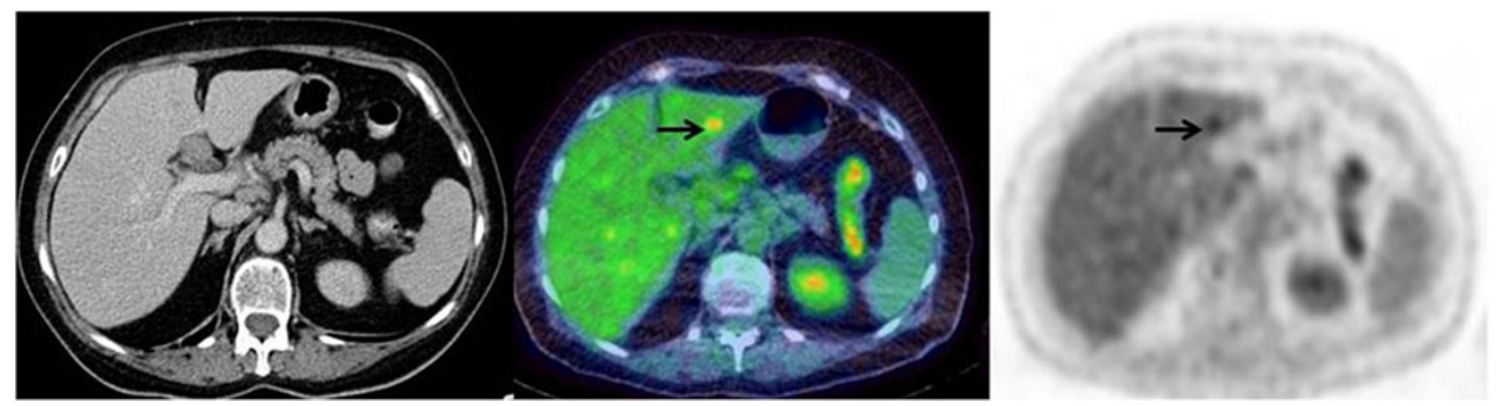

Fig. 1 Axial image panels of contrast-enhanced CT, fused PET-CT and PET in a patient with incidental gallbladder carcinoma in a cholecystectomy specimen. PET-CT showed uptake in a $10-\mathrm{mm}$ segment III liver metastasis (arrow) unsuspected on the initial contrast enhanced $\mathrm{CT}$ and subsequently confirmed with MRI 
represent disseminated malignancy. However, the patient had cholangitis with bile leak at the time of FDG PET-CT, as confirmed on subsequent staging laparoscopy.

FDG PET-CT was indeterminate in two cases, one with several enlarged lymph nodes showing low grade uptake and the other with mild FDG activity in a trace of pelvic free fluid.

Fourteen of the 62 confirmed primary cholangiocarcinomas were FDG negative on PET-CT, with an overall sensitivity of $77 \%$. Three of 39 intra-hepatic cholangiocarcinomas (SUV mean 14.4, range 3.7-45.8) and 11 of 23 extra-hepatic cholangiocarcinoma (SUV mean 6.9, range 4.4-18.5) were FDG negative, giving sensitivities of $92 \%$ and $52 \%$ respectively.

With regards to tumour morphology, 15 of the 62 confirmed primary cholangiocarcinomas were of the infiltrative type (6 of which were FDG negative giving a sensitivity of $60 \%$ ), whilst 47 were mass forming ( 8 of which were FDG negative giving a sensitivity of $83 \%$ ).

\section{Discussion}

Most previous studies examining the role of FDG PET-CT in biliary malignancy have focused on the effectiveness of FDG PET-CT in assessing the primary tumour and extent of any spread, with few considering its overall impact on patient management. This study aimed to evaluate the impact of FDG PET-CT on the management pathway of patients with primary biliary malignancy and to help define its role in the work-up of these tumours.

FDG PET-CT was concordant with findings on CT/MRI in 74 cases $(63 \%)-44$ with no distant disease on FDG PET$\mathrm{CT}$ or CT/MRI, 1 with indeterminate findings on both FDG PET-CT and CT/MRI and 29 with suspected distant disease on both FDG PET-CT and CT/MRI. Overall, FDG PET-CT had a major impact in $28 \%$ of cases $(33 / 118)$. It detected occult sites of disease in 24 patients, helped to characterise 7 indeterminate lesions on cross-sectional imaging and detected 2 incidental synchronous primary cancers.

FDG PET-CT had the biggest influence in the gallbladder carcinoma group, having a major impact in $39 \%$ of cases (12/31), including upstaging $27 \%$ of the scans performed for initial staging (7/26). FDG PET-CT changed management mainly by identifying occult sites of disease not detected by CT/MRI (in nine patients) and finding unsuspected sites of disease recurrence (in two patients). Few published studies describe rates of impact of FDG PET-CT in gallbladder cancer; however, our rate is somewhat higher than that reported previously. In a recent study by Corvera et al. [9], FDG-PET (without CT) altered management in $20 \%(25 / 126)$ of patients with biliary cancer, including $23 \%(7 / 31)$ of patients with gallbladder carcinoma. Similarly, Petrwosky et al. [10] in a study that included 14 patients with gallbladder carcinoma report a change of management in $17 \%$, whilst Lee et al. [11] in a study of 16 patients report a change of management in

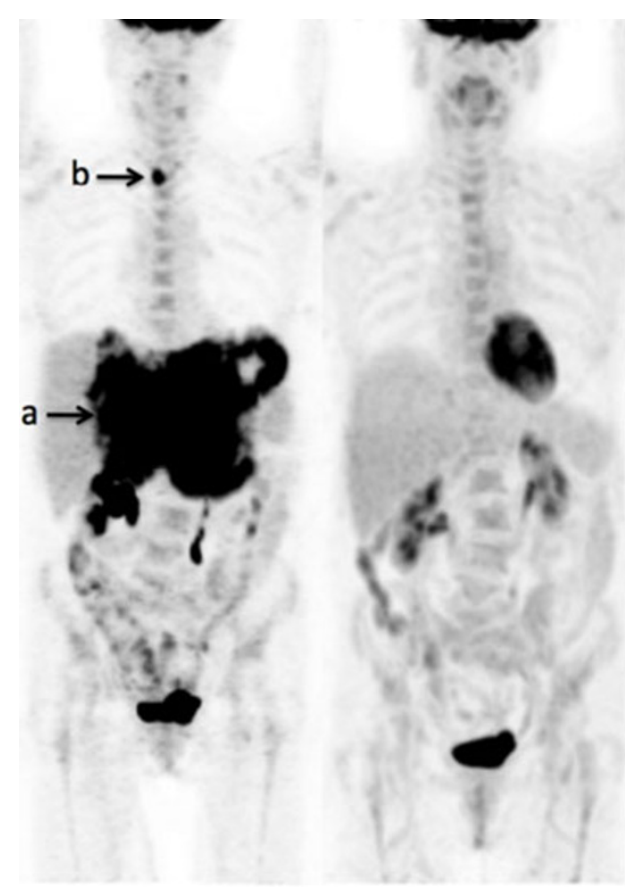

Fig. 2 PET maximum intensity projection (MIP) and axial image panels of CT and fused PET-CT in a patient with a large intra-hepatic cholangiocarcinoma. Initial PET-CT (upper row panel and left hand $M I P)$ showed FDG-avid primary tumour $(a)$ with unsuspected uptake in
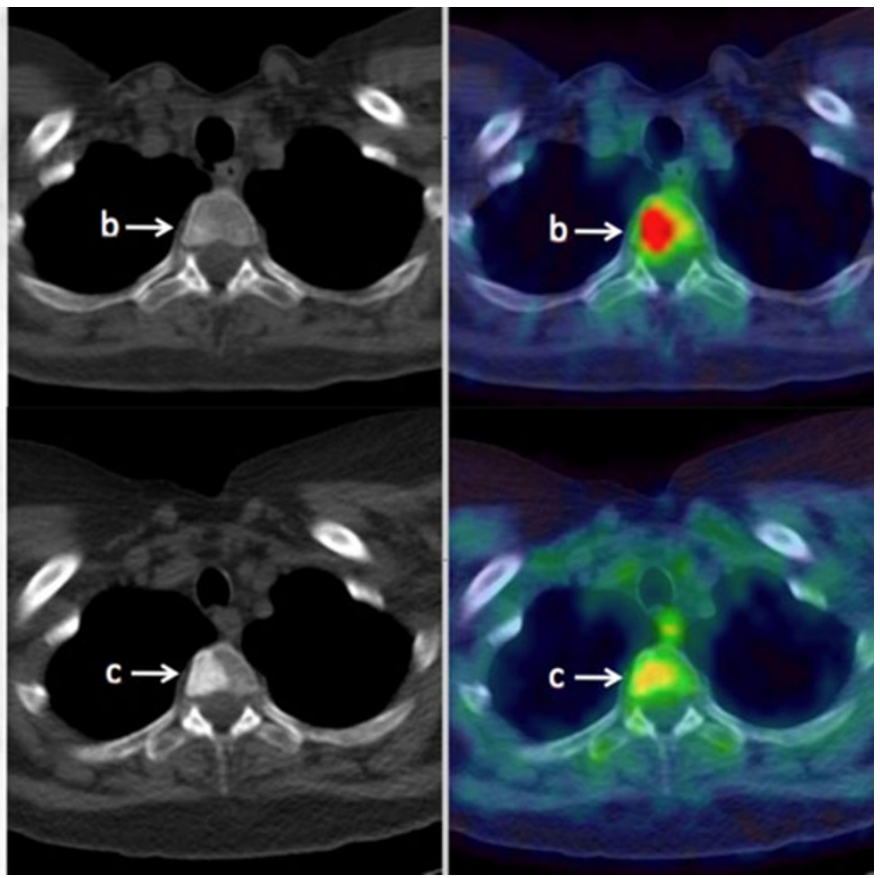

T2 vertebral body. PET-CT performed 6-months following chemotherapy (lower row panel and right hand MIP) showed mild residual uptake at $\mathrm{T} 2$ with sclerotic changes in the underlying bone in keeping with a healed/treated bone metastasis 

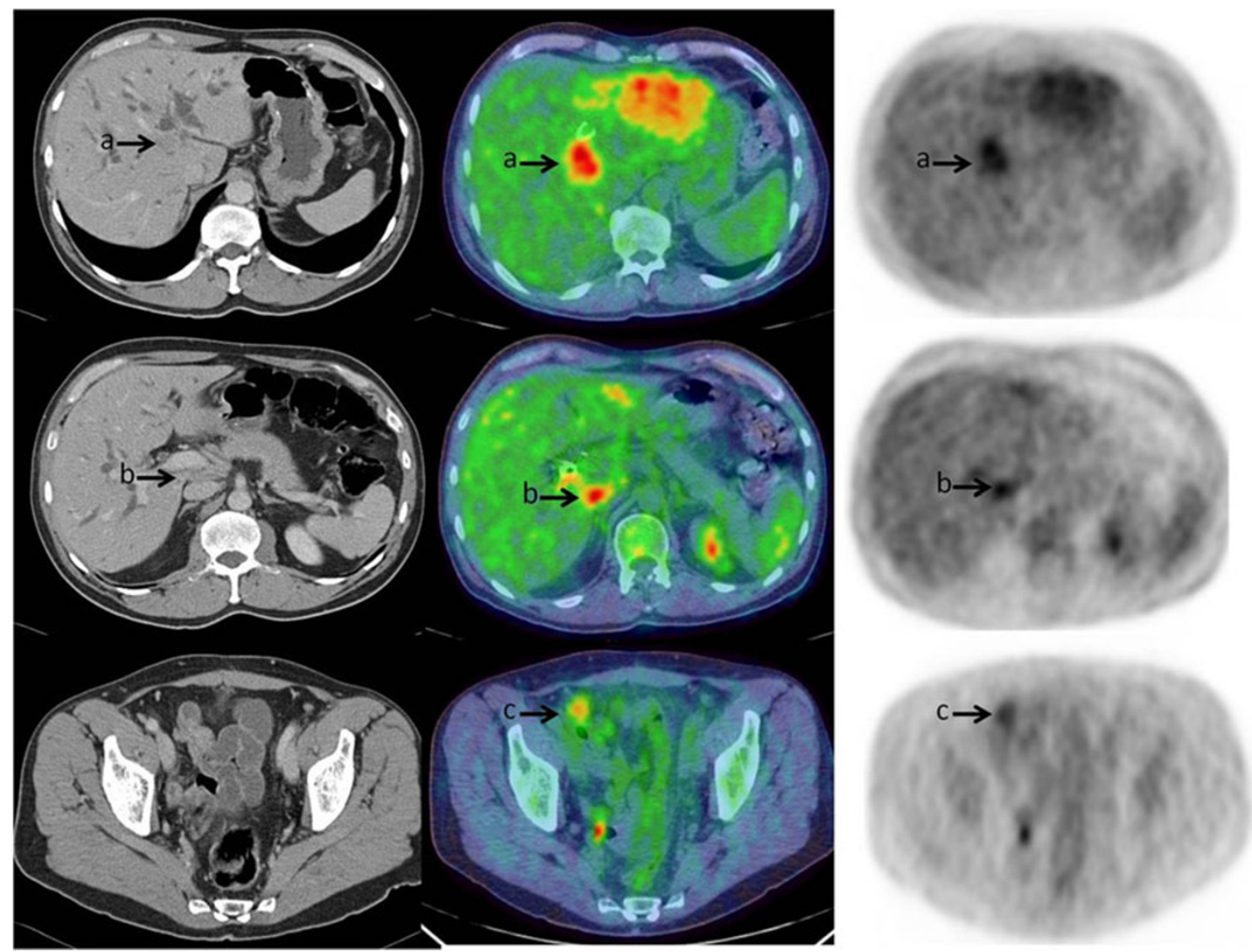

Fig. 3 Axial image panels of contrast enhanced CT (first column), fused PET-CT (second column) and PET (third column) in a patient with hilar cholangiocarcinoma. FDG-avid primary tumour $(a)$ with unsuspected nodal $(b)$ and peritoneal $(c)$ metastases. The peritoneal metastasis was not apparent on the contrast enhanced CT performed approximately 4 weeks prior to PET-CT
$9.8 \%$ of patients who were deemed resectable after standard staging; however both these studies also included patients with cholangiocarcinoma with no subgroup data. A possible explanation for the discrepancy in the rates of impact may be due to the process of patient selection. All patients in our study were individually selected for FDG PET-CT following discussion at a specialised MDT, where specific indications for performing the test were considered on a case-by-case basis. FDG PET-CT was not deemed necessary in some patients if conventional imaging with CT/MRI was thought to be clear-cut. Other published studies were less selective and included all patients with a suspected diagnosis of gallbladder carcinoma. The time lag to FDG PET-CT following conventional imaging may also have contributed to some patients with very aggressive tumours developing new sites of disease, which were then detected on FDG PET-CT. However, the vast majority of cases were scanned within a 90-day period with a mean time to PET-CT of 38 days. The other studies described did not report a time-frame for performing PET-CT and hence a comparison cannot be made.

Gallbladder carcinoma is associated with a rate of peritoneal metastasis as high as $30-75 \%$, and the risk of metastasis strongly correlates with the presenting $\mathrm{T}$ stage [12]. While FDG PET-CT can detect occult sites of disease not seen on conventional imaging, it may still fail to identify carcinomatosis due to reduced sensitivity in detection of sub-centimetre lesions (a recognised limitation of PET) and, in particular, small volume peritoneal disease. Three of the gallbladder cancers in our group thought resectable after FDG PET-CT were found to have unresectable peritoneal disease at laparotomy, with similar findings reported by others. In the study by Corvera et al. [9], five of the gallbladder cancers deemed resectable on PET were found to be unresectable at surgical exploration, three having locally advanced disease and two small volume peritoneal disease. Anderson et al. [13] in a study of 14 patients with gallbladder carcinoma detected peritoneal carcinomatosis in only three of six affected patients, attributing the results to the small size of the lesions.

One false-positive occurred in a patient with xanthogranulomatous cholecystitis (Fig. 4), a finding also reported by Wakabayashi et al. [14] and Koh et al. [15] both in eight patients with gallbladder carcinoma. Anderson et al. [13] described a false-positive in a patient studied 1 month after cholecystectomy with FDG uptake in residual post-surgical inflammatory change, thought to represent metastatic disease. Although this was not our experience, it does highlight the importance of considering the time interval between 
surgery and performing FDG PET-CT in order to minimise uptake in inflammatory tissue, especially when the gallbladder carcinoma is incidental and post-surgical staging is performed. In our experience, it is advisable to wait a minimum of 4 weeks and preferably at least 6 weeks following surgery to avoid false-positive uptake in post-surgical inflammation.

FDG PET-CT identified $100 \%$ of the 15 primary gallbladder carcinomas left in situ. This is consistent with previous published studies, which report sensitivity rates of 87.5-100\% for FDG PET-CT $[10,11]$ and $75-86 \%$ for PET alone $[9,13$, $15,16]$.

The overall sensitivity of FDG PET-CT for the detection of primary cholangiocarcinoma was generally lower at $77 \%$ (48/62), with a much higher sensitivity for intra-hepatic than for extra-hepatic cholangiocarcinoma $(92 \%, 36 / 39$ versus $52 \%, 12 / 23)$. Similar findings have been reported by
Petrowsky et al. [10] with FDG PET-CT sensitivity of $93 \%$ for intra-hepatic $(n=19)$ versus $55 \%$ for extra-hepatic cholangiocarcinoma $(n=36)$. Kim et al. [17] in a study of 123 patients with suspected cholangiocarcinoma also reported higher sensitivity of FDG PET-CT for intra-hepatic cholangiocarcinoma with PET-CT being falsely negative for the primary tumour in 15 patients (14 of which were extrahepatic cholangiocarcinomas).

As well as its location, the morphological characteristics of the primary tumour may also influence its detection rate. Similar to our results, Anderson et al. [13] in a study of 36 patients with suspected extra-hepatic cholangiocarcinoma reported a sensitivity of FDG-PET of $85 \%$ for nodular morphology (mass $>1 \mathrm{~cm}$ ) versus only $18 \%$ for infiltrating morphology. The difference has been attributed to infiltrating tumours having lower cellular density and causing little structural abnormality. Also, intra-hepatic cholangiocarcinomas
Fig. 4 PET maximum intensity projection (MIP) image, axial image panels of fused PET-CT, PET and contrast enhanced MRI demonstrating false-positive FDG uptake and abnormal gallbladder enhancement in a patient with suspected gallbladder carcinoma (arrows). Post-operative histology showed xanthogranulomatous cholecystitis with no evidence of malignancy

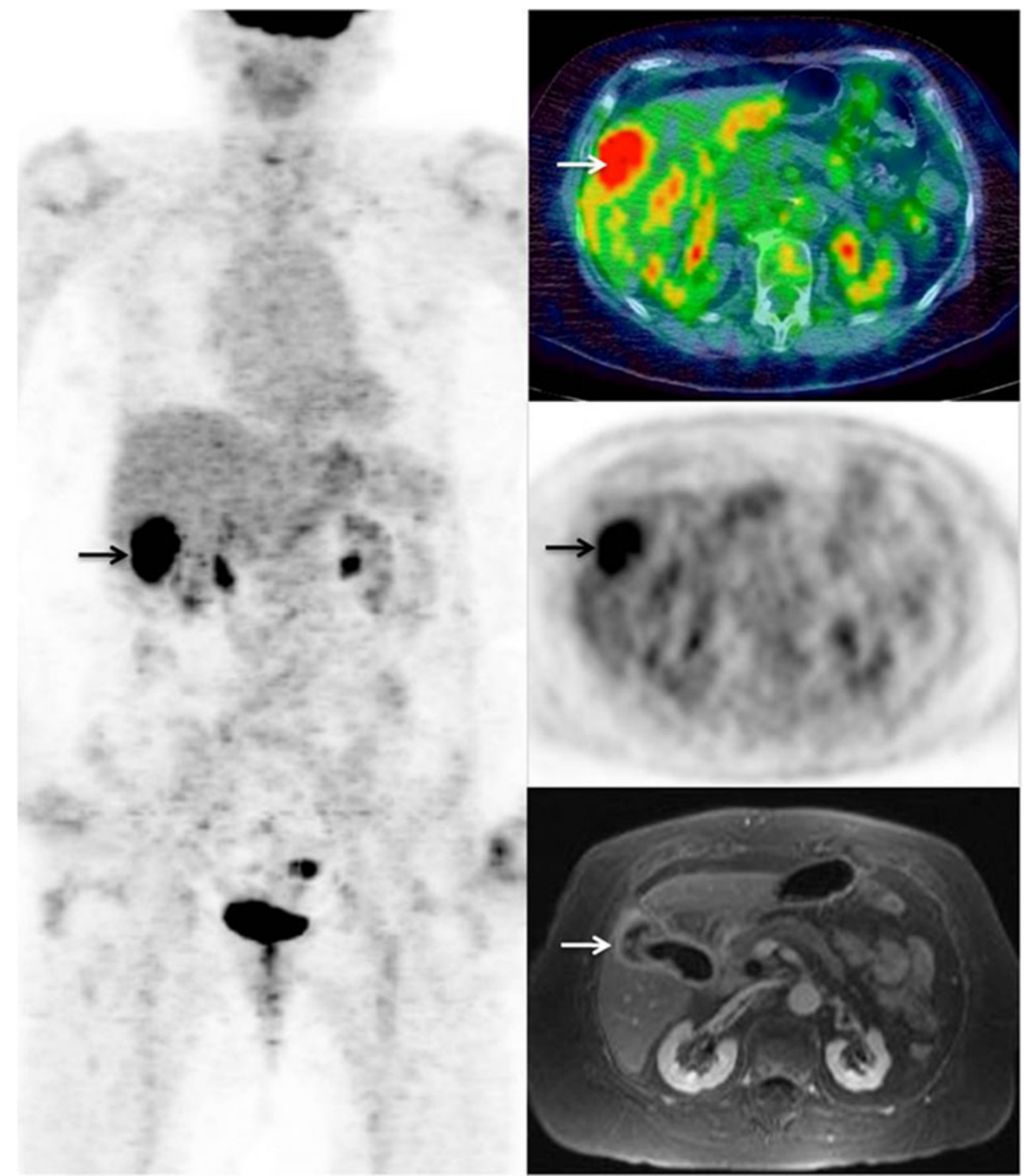


tend to be much larger at presentation. FDG PET-CT is known to have low sensitivity for mucinous tumours and hence may miss mucinous types of cholangiocarcinoma [18].

Whilst PET-CT may have little diagnostic advantage over traditional imaging modalities in detecting the primary cholangiocarcinoma [19], it has been shown to be substantially sensitive and specific for the detection and characterisation of metastases [20]. In this series, FDG PET-CT had a major impact on patient management in $24 \%(21 / 87)$ of cholangiocarcinomas, $26 \%$ (14/54) of intra-hepatic and $21 \%(7 / 33)$ of extra-hepatic tumours, finding occult sites of disease in 16 (including 5 cases of recurrence) and characterizing indeterminate lesions in another 5. Overall, FDG PETCT upstaged $19 \%(12 / 62)$ and downstaged $3 \%(2 / 62)$ of the scans performed for initial staging. This rate of impact is similar to that reported previously. In the study by Anderson et al. [13], FDG-PET led to a change in surgical management in $30 \%(11 / 36)$ of patients with suspected extra-hepatic cholangiocarcinoma due to detection of unsuspected metastases. Kim et al. [17] also reported about 94/123 patients who went on to have a final histological diagnosis of cholangiocarcinoma, for whom FDG PET-CT findings changed management in $15(15.9 \%)$, upgrading from resectable to unresectable in 7 patients $(7.4 \%)$ and downgrading a further $8(8.5 \%)$. Moon et al. [19], in a series of 54 patients, reported that FDG-PET (without CT) detected nine metastatic lesions not found by other imaging studies, stopping two patients from undergoing unnecessary laparotomy.

FDG PET-CT was falsely negative in two out of three patients with PSC, failing to detect the primary cholangiocarcinoma in both. One was a very small extra-hepatic tumour, which may have been below the size threshold for detection by PET. The lack of FDG uptake in the second tumour, a large well-differentiated intra-hepatic cholangiocarcinoma, is less clear. There are limited published data regarding the efficacy of FDG PET-CT in patients with PSC. Prytz et al. [21] performed FDG-PET on 24 patients with PSC awaiting liver transplantation with no evidence of malignancy on CT, MRI or ultrasound. Three patients had cholangiocarcinoma that was correctly identified by PET and the authors concluded that FDG-PET may be useful in screening for cholangiocarcinoma in patients with PSC. However, this did not prove to be the case in our experience or of others [22].

Similar to the gallbladder carcinoma group, FDG PET-CT was also falsely negative in three cholangiocarcinomas with unsuspected peritoneal disease found at laparotomy. In the study by Kim et al. [17], FDG PET-CT also failed to detect distant metastases in five patients, including four with peritoneal disease. We found a single false-positive in a patient with suspected peritoneal disease due to disseminated FDG uptake, however staging laparotomy was negative and appearances had been secondary to cholangitis and bile leak at the time of PET-CT. False-positive FDG uptake due to benign inflammatory causes is well recognised and a degree of caution has to be observed to avoid misinterpretation [23].

There are several limitations to this study which should be acknowledged. The referral mechanism for FDG PET-CT was determined on a case-by-case basis via a specialist MDT meeting, with each patient specifically chosen for the test to help answer a clinical or radiological ambiguity and therefore creating a selection bias. The time lag between studies also causes a potential bias for PET-CT superiority in patients with rapidly progressive disease. Another limitation is that data were collected retrospectively at a large tertiary referral centre, receiving patients from a wide geographical area. Consequently, a proportion of imaging studies were performed at referring regional hospitals using different equipment and acquisition protocols, possibly biasing image interpretation. The injection rate and volume of contrast medium were not patient tailored, which may also affect diagnostic accuracy. Assessment of impact was determined following a comprehensive review of all clinical and imaging data; however, such an assessment may be imperfect in a retrospective study.

Despite these issues, we found FDG PET-CT to be reliable in characterising indeterminate lesions and discriminating between benign and malignant disease with few false-positive results. The majority of false-negative findings were due to small volume peritoneal disease, which is a limitation for all imaging studies at present. FDG PET-CT was particularly effective at detecting metastatic disease that was unsuspected or equivocal on cross-sectional imaging studies, changing the stage of disease and influencing management in a significant number of patients. The rate of impact was higher in gallbladder carcinomas than in cholangiocarcinomas.

\section{Conclusion}

By detecting occult metastatic disease and characterizing indeterminate lesions, FDG PET-CT can have a major influence on clinical decision making in primary biliary malignancy both as part of initial work-up and for identifying recurrent disease, overall helping triage patients to the most appropriate treatment.

\section{Conflicts of interest None.}

Open Access This article is distributed under the terms of the Creative Commons Attribution License which permits any use, distribution, and reproduction in any medium, provided the original author(s) and the source are credited.

\section{References}

1. Alberts SR, Gores GJ, Kim GP et al (2007) Treatment options for hepatobiliary and pancreatic cancer. Mayo Clin Proc 82:628-637 
2. Gaujoux S, Allen PJ (2010) Role of staging laparoscopy in peripancreatic and hepatobiliary malignancy. World J Gastrointest 2:283-290

3. Weitz J, D'Angelica M, Jarnagin W, Gonen M, Fong Y, Blumgart L, Dematteo R (2004) Selective use of diagnostic laparoscopy prior to planned hepatectomy for patients with hepatocellular carcinoma. Surgery 135:273-281

4. Goere D, Wagholikar GD, Pessaux P et al (2006) Utility of staging laparoscopy in subsets of biliary cancers: laparoscopy is a powerful diagnostic tool in patients with intrahepatic and gallbladder carcinoma. Surg Endosc 20:721-725

5. Keidar Z, Haim N, Guralnik L, Wollner M, Bar-Shalom R, BenNun A, Israel O (2004) PET/CT using 18F-FDG in suspected lung cancer recurrence: diagnostic value and impact on patient management. J Nucl Med 45:1640-1646

6. Votrubova J, Belohlavek O, Jaruskova M et al (2006) The role of FDG-PET/CT in the detection of recurrent colorectal cancer. Eur J Nucl Med Mol Imaging 33:779-784

7. Bruzzi JF, Munden RF, Truong MT et al (2007) PET/CT of esophageal cancer: its role in clinical management. Radiographics 27:1635-1652

8. Hillner BE, Siegel BA, Shields AF et al (2008) Relationship between cancer type and impact of PET and PET/CT on intended management: findings of the National Oncologic PET Registry. J Nucl Med 49:1928-1935

9. Corvera CU, Blumgart LH, Akhurst T et al (2008) $18 \mathrm{~F}$ fluorodeoxyglucose positron emission tomography influences management decisions in patients with biliary cancer. J Am Coll Surg 206:57-65

10. Petrowsky H, Wildbrett P, Husarik DB et al (2006) Impact of integrated positron emission tomography and computed tomography on staging and management of gallbladder cancer and cholangiocarcinoma. J Hepatol 45:43-50

11. Lee SW, Kim HJ, Park JH et al (2010) Clinical usefulness of $18 \mathrm{~F}$ FDG PET-CT for patients with gallbladder cancer and cholangiocarcinoma. J Gastroenterol 45:560-566

12. Hueman MT, Vollmer CM Jr, Pawlik TM (2009) Evolving treatment strategies for gallbladder cancer. Ann Surg Oncol 16:21012115

13. Anderson CD, Rice MH, Pinson CW, Chapman WC, Chari RS, Delbeke D (2004) Fluorodeoxyglucose PET imaging in the evaluation of gallbladder carcinoma and cholangiocarcinoma. J Gastrointest Surg 8:90-97

14. Wakabayashi H, Akamoto S, Yachida S, Okano K, Izuishi K, Nishiyama Y, Maeta H (2005) Significance of fluorodeoxyglucosePET imaging in the diagnosis of malignancies in patients with biliary stricture. Eur J Surg Oncol 31:1175-1179

15. Koh T, Taniguchi H, Yamaguchi A, Kunishima S, Yamagishi $H$ (2003) Differential diagnosis of gallbladder cancer using positron emission tomography with fluorine-18-labeled fluoro-deoxyglucose (FDG-PET). J Surg Oncol 84:74-78

16. Rodríguez-Fernández A, Gómez Río M, Llamas Elvira JM et al (2004) Positron-emission tomography with fluorine-18-fluoro-2deoxy-D-glucose for gallbladder cancer diagnosis. Am J Surg 188:171-175

17. Kim JY, Kim MH, Lee TY et al (2008) Clinical role of 18F-FDG PET-CT in suspected and potentially operable cholangiocarcinoma: a prospective study compared with conventional imaging. Am J Gastroenterol 103:1145-1151

18. Jadvar H, Henderson RW, Conti PS (2007) [F-18]fluorodeoxyglucose positron emission tomography and positron emission tomography: computed tomography in recurrent and metastatic cholangiocarcinoma. J Comput Assist Tomogr 31:223-228

19. Moon CM, Bang S, Chung JB et al (2008) Usefulness of $18 \mathrm{~F}$ fluorodeoxyglucose positron emission tomography in differential diagnosis and staging of cholangiocarcinomas. J Gastroenterol Hepatol 23:759-765

20. Li J, Kuehl H, Grabellus F et al (2008) Preoperative assessment of hilar cholangiocarcinoma by dual-modality PET/CT. J Surg Oncol 98:438-443

21. Prytz H, Keiding S, Björnsson E, Broomé U, Almer S, Castedal M, Munk OL, Swedish Internal Medicine Liver Club (2006) Dynamic FDG-PET is useful for detection of cholangiocarcinoma in patients with PSC listed for liver transplantation. Hepatology 44:1572-1580

22. Fevery J, Buchel O, Nevens F, Verslype C, Stroobants S, Van Steenbergen W (2005) Positron emission tomography is not a reliable method for the early diagnosis of cholangiocarcinoma in patients with primary sclerosing cholangitis. J Hepatol 43:358-361

23. Culverwell AD, Scarsbrook AF, Chowdhury FU (2011) False positive uptake on 2-[ $\left.{ }^{18} \mathrm{~F}\right]$-fluoro-2-deoxy-D-glucose (FDG) positronemission tomography/computed tomography (PET/CT) in oncological imaging. Clin Radiol 66:366-382 